Juliana Bronzova

Cornelia M. van Duijn

Louis M. Havekes

Peter de Knijff

Christine Van Broeckhoven

Albert Hofman

\section{Apolipoprotein E genotype and concomitant clinical features in early-onset Alzheimer's disease}

Received: 8 June 1995

Received in revised form: 15 August 1995

Accepted: 3 October 1995

J. Bronzova $\cdot$ C. M. van Duijn (ه)

A. Hofman

Department of Epidemiology

\& Biostatistics and the Netherlands

Institute for Health Sciences,

Erasmus University Medical School,

Rotterdam, PO Box 1738 ,

NL-3000 DR Rotterdam, The Netherlands

Tel.: 31104087394

Fax: 31104365933

e-mail: duijn@epib.fgg.eur.nl

\section{J. Bronzova}

The Laboratory of Molecular Pathology, University Hospital of Obstetrics and Gynaecology, Medical University, Sofia, Bulgaria
L. M. Havekes · P. de Knijff

TNO, Institute of Prevention and Health, Gaubius Laboratory, Leiden, The Netherlands

C. Van Broeckhoven

The Neurogenetics Laboratory,

Flemish Institute of Biotechnology,

Born Bunge Foundation,

Department of Biochemistry,

University of Antwerp, Antwerpen,

Belgium

Abstract We have studied the relationship between the apolipoprotein E gene (APOE) and the development of myoclonus, tremors, rigidity and seizures in 168 patients with probable early-onset Alzheimer's disease (AD). There was a statistically significantly lower risk of tremor for carriers of the $\varepsilon 4$ allele of APOE. This allele was also associated with an increased risk of myoclonus. Our findings suggest that there may be differences in progression and clinical appearance in early-onset AD related to the APOE genotype.

Key words Alzheimer disease . Apolipoprotein E gene

\section{Introduction}

Alzheimer's disease (AD) is a clinically heterogeneous disorder. It is often accompanied by the development of extrapyramidal signs [13], myoclonus [8, 13] and seizures [8]. These concomitant clinical features in AD have been associated with a more rapid cognitive decline and increased mortality. Therefore, it has been suggested that these features may be markers of disease progression [13]. AD has been associated with apolipoprotein $\mathrm{E}$ (apoE) [14]. An increased risk of late-onset and early-onset $A D$ has been observed for carriers of the $\varepsilon 4$ allele of the apoE gene $\left(\mathrm{APOE}^{* 4}\right)$ compared with patients who carry two copies of the wild-type or normal allele $\varepsilon 3$ (APOE*3) $[3,14,15]$. The association with the $\varepsilon 2$ allele of the apoE gene (APOE*2) is less clear. Although a number of studies have reported a protective effect of the
$\mathrm{APOE}^{* 2}$ allele on the risk of $\mathrm{AD}[3,4]$, others have reported an increased $\mathrm{AD}$ risk for carriers of this allele [12, 16].

We have shown previously that the APOE gene is associated with survival of patients with early-onset AD [16]. Here we present a study on the role of the APOE gene in the development of concomitant clinical features in $\mathrm{AD}$. The aim of this analysis was to examine whether the $\mathrm{APOE}^{*} 4$ and $\mathrm{APOE}^{*} 2$ allele are associated with a distinct clinical expression.

\section{Patients and methods}

Patients were derived from a population-based study of early-onset AD. Details concerning the study design have been published elsewhere $[15,16]$. The protocol of the study has been reviewed by the medical ethics committee of the Erasmus University and Academic Hospital Dijkzigt. The series comprised all patients from two 
areas of the Netherlands, in whom the onset of AD was at age 65 years or earlier and who were diagnosed as having $\mathrm{AD}$ in the period January 1980 to July 1987 . The diagnosis of probable earlyonset $\mathrm{AD}$ was verified according to a standard protocol similar to NINCDS-ADRDA criteria. Information was available for 198 $(99 \%)$ of the 201 patients who met the inclusion criteria. Informed consent was obtained from all patients. For all patients, detailed data on family history of dementia in first-, second- and third-degree relatives were collected [15]. These data were always verified by a sibling of the patient. The family history of subjects with no first-degree relatives with dementia was classified as negative and of those with at least one first-degree relative with dementia as positive. APOE typing could be performed in $175(88 \%)$ of the patients [15].

At entrance into the study, data on medication and on the occurrence of clinical features including myoclonus, tremor, rigidity and seizures were collected through medical records from the general hospital where the patient was diagnosed and from the nursing home for those patients that were institutionalized. At follow-up in 1990, medical records from general hospitals and nursing homes were up-dated for all patients. The protocol of data collection and the relationship of the clinical features to mortality have been described elsewhere [11]. The present analysis is based on the presence of clinical features at diagnosis or their occurrence during the follow-up of the patients up to 1990 . We have considered only clinical features that were not related to medication. The mean period of follow-up after diagnosis was 6 years (range $2-11 ; \mathrm{SD}=2$ ). Data on the clinical course were available for $168(96 \%)$ of the 175 patients in whom APOE was typed.

All statistical analyses were carried out with the Epidemiological Graphics Estimation and Testing package (EGRET). To adjust for censoring of the data which occurred because not every patient was followed until death, Cox's proportional hazards model was used to estimate the effect of the APOE gene on the occurrence of a clinical feature [6]. In addition, we have adjusted all analyses for left truncation, which occurred because not all patients were included in our study at the time of diagnosis [10].

\section{Results}

General characteristics of the 168 patients are given in Table 1 for APOE*3 homozygotes (APOE3E3), APOE*4 and $\mathrm{APOE}^{*} 2$ carriers. Among APOE*2 carriers, there were significantly $(P<0.05)$ more men $(50 \%)$ compared with APOE*4 $(31 \%)$ and APOE3E3 (30\%) carriers. A family history of dementia was found more often among carriers of the APOE*4 allele $(P<0.05)$. APOE genotype was not associated with age at onset. Also, the score on the Clinical Dementia Rating (CDR) scale [9] at diagnosis was not associated with the APOE $* 4$ allele [relative risk $=0.7 ; 95 \%$ confidence interval (CI) 0.3-1.5; reference APOE3E3] or the $\mathrm{APOE}^{*} 2$ allele (relative risk $=1.1 ; 95 \%$ CI $0.3-3.3$; reference $A P O E 3 E 3)$.

Table 2 shows the risk of concomitant clinical features by APOE genotype. APOE*4 carriers had a significant, 2.2-fold higher risk of myoclonus compared with carriers of the APOE3E3 genotype (95\% CI: 1.1-4.4). A non-significant increase in risk of myoclonus was found for APOE*2 carriers. APOE*4 carriers had a significantly lower risk of tremors. No significant association was observed between the APOE genotype and the risk of rigidity and seizures. The risk of myoclonus and tremors by
Table 1 Characteristics of the 168 patients with early-onset $\mathrm{Alz}$ heimer's disease (AD)

\begin{tabular}{|c|c|c|c|}
\hline Clinical feature & $\begin{array}{l}\text { APOE3E3 } \\
n=57\end{array}$ & $\begin{array}{l}\mathrm{APOE}^{*} 4 \\
\text { carriers }^{\mathrm{a}} \\
n=88\end{array}$ & $\begin{array}{l}\mathrm{APOE}^{*} 2 \\
\text { carriers }^{\mathrm{a}} \\
n=24\end{array}$ \\
\hline Men & $17(30 \%)$ & $27(31 \%)$ & $12(50 \%)$ \\
\hline Family history positive & $29(51 \%)$ & $61(69 \%)$ & $12(50 \%)$ \\
\hline Mean onset age (SD) & $57 \quad(6)$ & $58(6)$ & $56 \quad(6)$ \\
\hline
\end{tabular}

a One subject with the APOE2E4 genotype is included as a $\mathrm{APOE}^{*} 4$ as well as an $\mathrm{APOE} * 2$ carrier

Table 2 The relative risk (95\% confidence interval) of myoclonus, tremor, rigidity and seizures in early-onset $\mathrm{AD}$ for $\mathrm{APOE}^{*} 4$ and $\mathrm{APOE}^{*} 2$ carriers compared with the risk for carriers of the APOE3E3 genotype

\begin{tabular}{llll}
\hline Clinical feature & APOE3E3 & APOE*4 $^{*}$ & APOE*2a \\
\hline Myoclonus & 1 & 2.2 & 1.9 \\
& Reference & $(1.1-4.4)$ & $(0.8-4.9)$ \\
& $n=14^{\mathrm{b}}$ & $n=23$ & $n=9$ \\
Tremor & 1 & 0.4 & 1.4 \\
& Reference & $(0.2-0.8)$ & $(0.6-3.3)$ \\
& $n=14$ & $n=21$ & $n=7$ \\
Rigidity & 1 & 0.7 & 0.8 \\
& Reference & $(0.4-1.3)$ & $(0.4-1.9)$ \\
& $n=22$ & $n=31$ & $n=9$ \\
Seizures & 1 & 0.8 & 1.1 \\
& Reference & $(0.5-1.4)$ & $(0.5-2.2)$ \\
& $n=30$ & $n=42$ & $n=12$ \\
\hline
\end{tabular}

a One subject with the APOE2E4 genotype is included as a $\mathrm{APOE}^{*} 4$ as well as an $\mathrm{APOE} * 2$ carrier

b The number of patients who developed the clinical feature over time

APOE genotype is plotted in Fig. 1. Although at each point in time after diagnosis the risk of myoclonus was higher in $\mathrm{APOE}^{*} 4$ and $\mathrm{APOE} * 2$ carriers than in APOE3E3 carriers, in the 11 years of follow-up $55 \%$ (95\% CI: $35 \%$ $78 \%$ ) of the APOE3E3 carriers developed myoclonus. After 3 years of follow-up, the risk of tremors was consistently lower in $\mathrm{APOE}^{*} 4$ than in $\mathrm{APOE}^{*} 2$ and APOE3E3 carriers.

When stratifying for family history of dementia, the association of the $\mathrm{APOE}^{*} 4$ and $\mathrm{APOE}^{*} 2$ allele with myoclonus was strongest in those with a negative family history of dementia. The risk of myoclonus was $2.8(95 \% \mathrm{CI}$ : 1.0-7.3) times greater for APOE* 4 carriers and 2.3 (95\% CI: $0.7-7.3)$ times greater for $\mathrm{APOE}^{*} 2$ carriers when compared to APOE3E3 carriers (not in table). Among those with a positive family history, there was a 1.5 -fold (95\% CI: 0.6-3.8) increased risk associated with the $\mathrm{APOE}^{*} 4$ allele and a 1.4 -fold $(95 \% \mathrm{CI}: 0.4-5.8)$ increase with the APOE* 2 allele. There was no evidence for effect modification after stratifying the data by gender. 

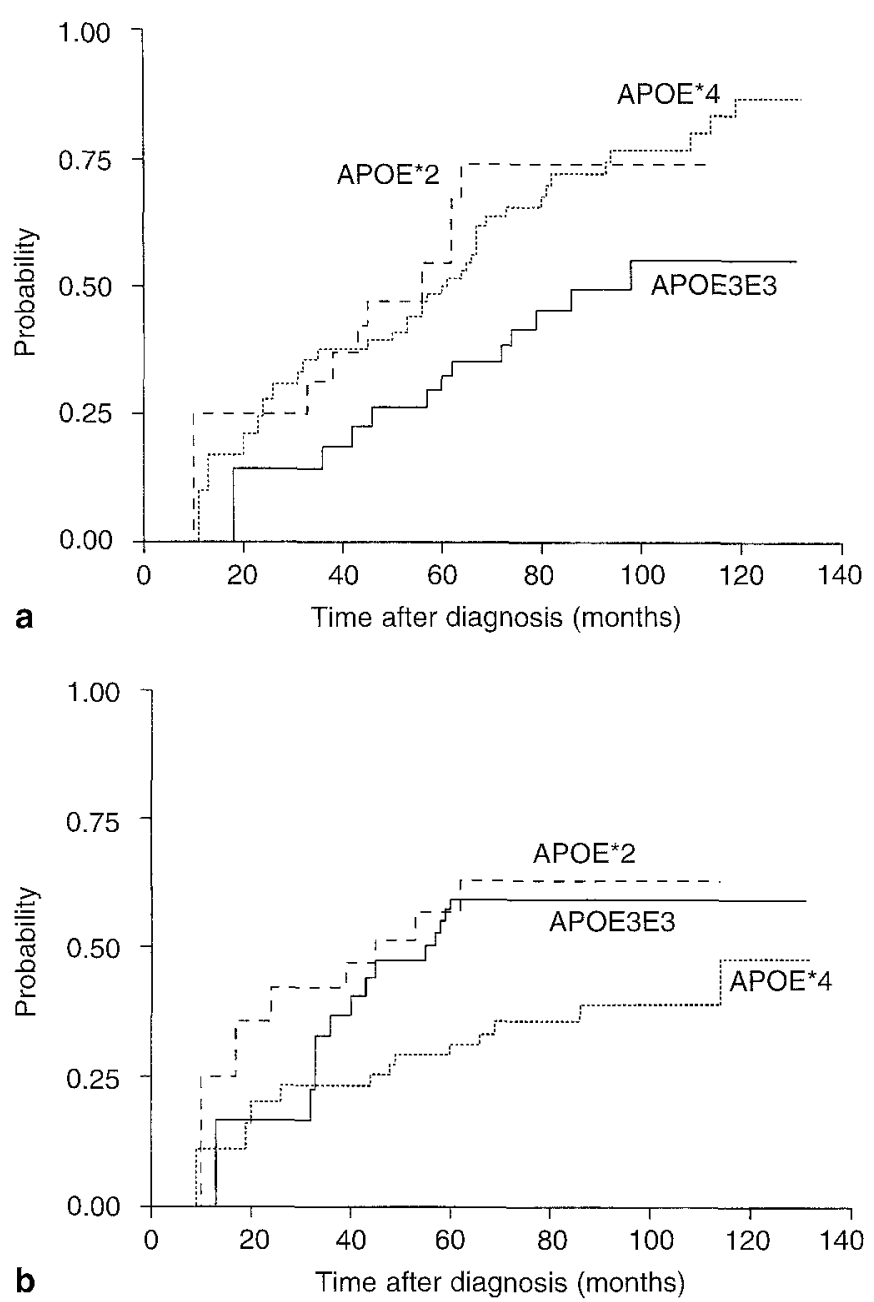

Fig. 1 The risk of myocionus (a) and tremors (b) by APOE genotype

\section{Discussion}

In this study the occurrence of extrapyramidal signs, myoclonus and seizures was assessed through medical records. The diagnosis of the clinical features was therefore not standardized and their occurrence may not always have been recorded. To overcome this problem, we limited our study to those features that required special medical and nursing care and consequently are well diagnosed and most likely reported in the medical records. In addition, we relied on neurological examinations by trained neurologists as well as by nursing home physicians. Although in the Netherlands the latter perform neurological examinations routinely at admission of an AD patient to the nursing home, the reliability of their diagnosis may vary considerably. However, we have previously shown the occurrence of extrapyramidal signs, tremors and rigidity, to be strongly associated $(P=0.001)$ while neither tremors nor rigidity were associated with the occurrence of myoclonus or seizures [11]. This suggests that tremor, one of the features associated with APOE, was suitable distinguished by the participating physicians from the other associated feature, myoclonus. Also, it is important to note that in order to bias our results the misclassification in the clinical features must have occurred differentially in APOE3E3, $\mathrm{APOE} * 4$ and $\mathrm{APOE} * 2$ carriers in order to introduce an artificial relationship. It is unlikely that this has happened systematically, as the nursing home staff were not aware of the patient's APOE genotype. Most likely, bias due to misdiagnosis has occurred randomly and independent of the APOE genotype in our study. The effect of such misclassification is most likely that existing relationships are diminished rather than nonexisting associations being created.

Our findings suggest that there may be differences in the clinical course of early-onset AD related to the APOE genotype. One earlier study failed to show an association [1]. However, that study was based on late-onset AD and the number of patients was small $(n=60)$ while the follow-up period was limited (3 years) [1]. Our study shows that $\mathrm{APOE}^{*} 4$ carriers have a significantly decreased risk of developing tremors. Although there is considerable controversy concerning the role of APOE in the survival of AD patients [2,5], our study and those of others suggest that the APOE*4 allele is associated with a slower progression of $\mathrm{AD}$ and a reduced risk of mortality $[1,7$, 16]. Our earlier finding of a lower mortality for early-onset $\mathrm{AD}$ patients who carry the APOE*4 allele [16] agrees with the lower risk of tremors, because the latter probably indicate an advanced stage of AD [13]. There are two possible explanations for these findings. Firstly, the diagnosis of AD occurs earlier in $\mathrm{APOE}^{*} 4$ carriers than in other patients, leading to an artificially prolonged survival. Secondly, the APOE*4 allele is truly associated with a slow progression of $\mathrm{AD}$. The first explanation is plausible, e.g. because $\mathrm{APOE}^{*} 4$ carriers are more likely to have a positive family history [15] or a history of cardiovascular disease and may be more alert when initial symptoms appear. However, in our population the age of onset and the severity of the dementia at diagnosis as measured by the CDR score was similar in patients with and without the APOE*4 allele [15]. Adjusting the analysis for the CDR score at diagnosis or the duration of dementia at diagnosis did not change any of our conclusions, suggesting that the explanation of a relatively early diagnosis in APOE* $^{*}$ carriers is unlikely. In addition, our finding that the risk of myoclonus was significantly increased for $\mathrm{APOE}^{*} 4$ carriers challenges this hypothesis, since the occurrence of both tremors and myoclonus has been shown to indicate advanced stages of AD [13].

The increased risk of myoclonus for $\mathrm{APOE}^{*} 4$ carriers cannot be explained by disease progression but suggests an unusual early expression of myoclonus in early-onset AD patients with the APOE*4 allele. The hypothesis of a distinct clinical appearance of patients is further sup- 
ported by the finding that the association was strongest in those with a negative family history. It is important to note that the occurrence of myoclonus was not limited to APOE*4 carriers. APOE3E3 carriers may also develop myoclonus, albeit at a later stage. The finding that carriers of the APOE*2 allele have a risk of tremors similar to that of carriers of the APOE3E3 genotype, while at the same time the risk of myoclonus for $\mathrm{APOE}^{*} 2$ carriers is similar to that for $\mathrm{APOE}^{*} 4$ carriers, seems contradictory. However, our findings on the APOE* 2 allele are based on a small number of patients.

This is the first study to suggest that there may be differences in progression and clinical appearance in earlyonset AD related to the APOE genotype. An important advantage of our study is the population-based design in which patients have been followed for an extended period. However, our findings are based on a specific subgroup of
$\mathrm{AD}$ patients with an early onset of $\mathrm{AD}$. Further, we have not collected data on the occurrence of the features under study by a standardized clinical examination. Our findings therefore remain to be confirmed by other studies on the clinical course of AD.

Acknowledgements This study was supported by the Netherlands Organization for Scientific Research (NWO), the Netherlands Institute for Health Sciences (NIHES), the Eurodem EC Concerted Action on dementia, the Flemish Biotechnology Program and the Belgian National Fund for Scientific Research (NFSR). C.V.B. is a research associate of the NFSR Belgium. We thank Drs. Wim Schulte, Teun Tanja, Rob Haaxma, Arie Lameris, Rolf Saan for assisting with case diagnosis and Helen de Bruijn, Micheline de Haes, Jeanette Kamman, Hilda Kornman, Hanneke van Meurs and Caroline Valkenburg for their help in data collection. Hans van der Boom, Marc Cruts, Anita Wehnert and Joke De Voecht are thanked for apolipoprotein E typing.

\section{References}

1. Basun H, Grut M, Winblad B, Lannfelt L (1995) Apolipoprotein $\varepsilon 4$ allele and disease progression in patients with late-onset Alzheimer's disease. Neurosci Lett 183:32-34

2. Bennett C, Crawford F, Osborne A, Diaz P, Hoyne J, Lopeze R, Roques P, Duara R, Rossor M, Mullan M (1995) Evidence that the APOE locus influences rate of disease progression in late onset familial Alzheimer's disease but is not causative. Am J Med Genet 60:1-6

3. Chartier-Harlin MC, Parfitt M, Legrain S, Pérez-Tur J, Brousseau T, Evan A, Berr C, Vidal O, Roques P, Gouriet V, Fruchart J-C, Delacourte A, Rossor M, Amouyel P (1994) Apolipoprotein E, $\mathrm{E} 4$ allele as a major risk factor for sporadic early and late-onset form of Alzheimer's disease: analysis of the 19q13.2 chromosomal region. Hum Mol Genet 3:569-574

4. Corder EH, Saunders AM, Risch NJ, Strittmatter WJ, Schmechel DE, Gaskell PC, Rimmler JB, Locke PA, Conneally PM, Schmader KE, Small GW, Roses AD, Haines JL, PericakVance MA (1994) Protective effect of apolipoprotein $E$ type 2 allele for late onset Alzheimer disease. Nature Genet $7: 180-184$
5. Corder EH, Saunders AM, Strittmatter WJ, Schmechel DE, Gaskell PC, Rimmler JB, Locke PA, Conneally PM, Schmader KE, Tanzi RE, Gusella JF, Small GW, Roses AD, PericakVance MA, Haines JL (1995) Apolipoprotein $\mathrm{E}$, survival in Alzheimer's disease patients, and the competing risk of death and Alzheimer's disease. Neurology $45: 1323-1238$

6. Cox DR (1972) Regression models and life tables. J R Stat Soc 34:187-220

7. Frisoni GB, Govoni S, Geroldi C, Bianchetti A, Calabresi L, Franceschini G. Trabucchi M (1995) Gene dose of the $\varepsilon 4$ allele apolipoprotein $\mathrm{E}$ and disease progression in sporadic late-onset Alzheimer's disease. Ann Neurol 37:596-604

8. Hauser WA, Morris ML, Heston LL, Anderson VE (1986) Seizures and myoclonus in patients with Alzheimer's disease. Neurology 36:1226-1230

9. Hughes CP, Berg L, Danziger WL, Colen LA, Martin RL (1982) A new clinical scale for the staging of dementia. Br J Psychiatry 140:566-572

10. Kurtzke JF (1989) On estimating survival; a tale of two censors. J Clin Epidemiol 42:169-175

11. Samson WN, Van Duijn CM, Hop WCJ, Hofman A (1996) Clinical features and mortality in patients with early-onset Alzheimer's disease. Eur Neurol 36: 103-106

12. Sorbi S, Nacmias B, Forleo P, Latorraca $S$, Gobbini I, Bracco L, Piacentini S, Amaducci L (1994) ApoE allele frequencies in Italian sporadic and familial Alzheimer's disease. Neurosci Lett 177:100-102
13. Stern Y, Albert M, Brandt J, Jacobs DM, Tang MX, Marder K, Bell K, Sano M, Devanand DP, Bijlsma F (1994) Utility of extrapyramidal signs and psychosis as predictors of cognitive and functional decline, nursing home admission, and death in Alzheimer's disease: prospective analyses from the Predictors Study. Neurology 44:2300-2307

14. Strittmatter WJ, Saunders AM, Schmechel D, Pericak-Vance M, Enghild J, Salvesen GS, Roses AD (1993) Apolipoprotein E: high avidity binding to beta-amyloid and increased frequency of type 4 allele in late-onset familial Alzheimer's disease. Proc Natl Acad Sci USA 90:1977-1981

15. Van Duijn CM, Knijff $P$ de, Cruts $M$, Wehnert A, Havekes LM, Hofman A, Van Broeckhoven C (1994) Apolipoprotein $\mathrm{E} 4$ allele in a population-based study of early-onset Alzheimer's disease. Nature Genet 7:74-78

16. Van Duijn CM, Knijff $P$ de, Wehnert A, De Voecht J, Bronzova JB, Havekes LM, Hofman A, Van Broeckhoven C (1995) The apolipoprotein $E \varepsilon 2$ allele is associated with an increased risk of early-onset Alzheimer's disease and a reduced survival. Ann Neurol 37:605610 